There are some important points to this letter, however, particularly regarding what is referred to as the non-medicalisation of bereavement.

We are ignoring our duty as professional carers if we do not concern ourselves with the imminently bereaved and are just brushing under the carpet something we find difficult to deal with by withdrawing from our responsibility to bereaved loved ones. We invite relatives, without coercion or callousness, into the resuscitation room and give information in a compassionate way. Infringement of autonomy occurs when a relative or patient is deprived of the information he or she needs for autonomous decision making. It is unacceptable medical paternalism to withhold that information in the fear that it will cause the patient or relative harm.

1 Mitchell MH, Lynch MB. Should relatives be allowed in the resuscitation room? J Accid Emerg Med 1997;14:366-9.

2 Adams S, Whitlock M, Higgs $R$, et al. Should relatives be allowed to watch resuscitation? $\mathrm{BMJ}$ 1994;308:1687-92.

3 Hanson C, Strawser D. Family presence during cardiopulmonary resuscitation: Foote Hospital emergency department's 9 year perspective. J Emerg Nurs 1992;18:104-6.

\section{Anterior glenohumeral dislocation}

EDrToR,-I would like to make a few comments on the very informative article by $A$ Gleeson on shoulder dislocation. ${ }^{1}$

I am one of the many people who reduce shoulder dislocation without injections of drugs but I do not choose this method for physician convenience. It is possible to achieve pain free reduction of the dislocation within a few minutes of the patient's arrival in the accident and emergency (A\&E) department without the use of injected drugs. It simply needs explanation, reassurance, a quiet room, use of a simple relaxation or hypnosis technique, and gentle manipulation.

Relaxation reduces muscular spasm and therefore pain. Entonox given before manipulation promotes relaxation.

The fact should be emphasised that most dislocations can be reduced without use of force and that traction is not necessary. The patient should be informed of this as it helps them to relax. It may take 10 minutes of slow movement to achieve reduction but in a relaxed and confident patient this procedure can be pain free.

It also means that an early attempt at reduction can be made before radiography is considered: there are no complications of slow gentle movement. If the patient is clearly in discomfort then the attempt is stopped and the traditional method used.

I would like to point out that $\mathrm{D}$ and $\mathrm{E}$ in fig 2 are in the wrong order.

Internal rotation is not part of the manoeuvre itself: it simply puts the arm into the position where it can be immobilised. If there is no resistance to internal rotation across the chest then reduction has been achieved. If there is resistance and pain then the dislocation persists.

Can I suggest that the gold standard for shoulder dislocation is reduction:

- Without undue pain

- Without injections of potent drugs

- As soon as possible after arrival in the A\&E department

- Without further damage because force is not used

- Without the complications of benzodiazepines or opiates necessitating admission
This is achievable: my own experience is that at least half of shoulder reductions can be done this way once the basic techniques become familiar. The success rate improves with practice, familiarity, and confidence.

I wholeheartedly endorse the view that reduction of shoulder dislocation should be a distress free experience and that force should not be involved but disagree that "gold standard" means use of potent drugs.

ALAN LANNIGAN

Accident and Emergency Department, Crosshouse Hospital, Kilmarnock KA1 OBE

1 Gleeson AP. Anterior glenohumeral dislocation: what to do and how to do it. J Accid Emerg Med $1998 ; 15: 7-12$

\section{The author replies}

I agree with Mr Lannigan that it is indeed possible to achieve a relatively pain free reduction of a dislocation within a few minutes of the patient's arrival in $A \& E$ without the use of injected drugs by encouraging the patient to relax and to avoid sudden painful movements of the arm during manipulation. As a minimum though, I would recommend that all patients should be offered a trial of Entonox which will certainly facilitate the reduction process in the majority of cases. It is probably unwise to attempt reduction of an anterior glenohumeral dislocation without prior radiography as there may be a medicolegal battle over whether a fracture evident on a postreduction film was present before reduction or occurred as a result of the reduction manoeuvre. The only circumstance where I would reduce an anterior dislocation without prior radiography would be in recurrent dislocators who have not fallen onto the arm and who sustained the dislocation with a combination of simple abduction and external rotation.

\section{Do we need to be propped up with protocols?}

EDrToR,-Stedman's Medical Dictionary defines protocols as ..."a precise and detailed plan for the study of a biomedical problem or for a regimen of therapy". ' Clinicians argue for and against protocols but seemingly their number is increasing every day. We wondered how many protocols could be drawn up for an accident and emergency (A\&E) department. Using the index of the Cambridge Textbook of Accident and Emergency Medicine as a list, ${ }^{2}$ we selected protocols based on symptoms, signs, diagnoses, and therepaeutic modalities relevant to British A\&E departments.

We excluded conditions which are rare and not serious, administrative and local protocols, tropical diseases, and specialist conditions, for example Salter-Harris fracture classifications. We did not subdivide every major diagnosis, for example atrial fibrillation was not divided into acute and chronic. We did not consider protocols for the patient without an established diagnosis.

Four hundred and two potential protocols were identified of which 280 were deemed essential for every department.

While protocols may enhance quality of care, save time, make medical practice more focused and scientific and reduce litigation, they may also produce more "cookbook" type medicine, inhibit free thinking, and make deviation from protocols open to litigation. Will delay in initiating treatment due to protocol consultation not jeopardise a patient's critical condition? Will it be possible for a jun- ior doctor, or even a senior one, starting in the A\&E department to study and remember all protocols and practise accordingly? By practising protocol based care in the A\&E department are we not admitting that that training or experience or supervision (or all three) are lacking?

The results of our survey, because of the large numbers of protocols involved, suggest protocol based emergency care will be difficult. It is therefore even more important that training is improved and supervision readily available in $\mathrm{A} \& \mathrm{E}$ departments.

F KHAN

D HUGHES

B MCNICHOLL

Accident and Emergency Department, Royal Hospitals, Grosvenor Road,

Belfast BT12 6BA

1 Stedman's medical dictionary. Baltimore: Williams and Wilkinson, 1995: 1446.

2 Skinner D, Swain A, Peyton R, et al. Cambridge textbook of accident and emergency medicine. textbook of accident and emergency medicine. 251-1271.

\section{CS "gas" is not a gas}

EDITOR,-The case report by Breakell and Bodiwala usefully highlights several dangers of CS. ${ }^{1}$ However, by constantly referring to the agent as a gas they reduce the logic of many of their arguments. CS is a solid (melting point $94-95^{\circ} \mathrm{C}$ ), though it may behave as a gas when dispersed as a fine powder from a pyrotechnic device. In Britain, however, CS is deployed dissolved in a solvent. When the solvent has evaporated, solid crystals of CS remain and may be deposited on the skin. This is usually the situation when the patient reaches hospital. Fanning with air, wiping exposed areas with dry tissue, and removal of clothing will be the most effective remedies. Clearly this would not be appropriate or adequate decontamination after exposure to a gas. By using the term CS and not calling it CS gas, people will understand the logic behind the emergency care of exposed victims.

JOHN HENRY

Department of Accident and Emergency Medicine, St Marys Hospital, Praed Street, London W2 $1 \mathrm{NY}$

\section{The authors reply}

We agree entirely with Professor John Henry's comments that CS "gas" is not a gas, and reference to this fact was made in the discussion. ${ }^{1}$ We used the term CS gas due to the popularity and widespread use in the medical literature, not as a description of its chemical properties. We made the point that fanning with air (electric fan) caused contamination of the accident and emergency department and CS powder (crystals) converting to a solution on the surface of the eye should be managed by eye irrigation rather than air currents. ${ }^{2}$

1 Breakell A, Bodiwalwa GG. CS gas exposure in a crowded night club: the consequences for an accident and emergency department. J Accid

2 Yih JP. CS gas injury to the eye. BMJ 1995;311: 276 .

\section{Fire}

EDIToR,-On Sunday 5 October 1997 at 3.00 pm we were working in our emergency department at the Derbyshire Royal Infirmary, Derby. The fire alarm sounded and we completely evacuated the department into the car park. We quickly sent one very ill patient to 
the intensive care unit, and another from the computed tomography scanner to the neurosurgeons in Nottingham.

The fire brigade came and informed us of a fire in the basement under the department. The department was full of smoke and we could not work in it for several hours.

Several patients were sent directly to wards and the ambulance service diverted patients to other hospitals. We opened the outpatient department and the day case unit and treated casualties there. This makeshift plan was difficult to implement. A few hours later when the fire was put out and the smoke dispersed, we returned with our patients to an undamaged department.

Before this experience we have had two fires started deliberately by patients in the department-one in a toilet and one in a waiting room. So the possibility of this, or indeed any, emergency department being unable to function for a period of time is always there. Fire is the most likely cause but explosions, building collapse, electrical failure, flooding, and violent affrays are others. Working in over 20 hospitals over the last 35 years, AF-M has seen all of these, but only the smoke from this fire was serious enough to cause our emergency department to be closed.)

We had no local plan in our hospital to deal with this contingency. We do now have such a plan and I am sure that other hospitals should follow our example to avoid being caught out.

There are no national guidelines for hospitals or accident and emergency departments to close, but the ambulance service has national guidelines for diverting all ambulances to other hospitals in circumstances such as these. This can easily be arranged through your local ambulance service.

A mobile field hospital could in theory be provided by the armed services. This requires quite a fair sized space to set it up. Reliable sources with experience of setting up these hospitals have told us that this takes at least 48 hours. So this is not a "quick" option, but an alternative if the emergency department is out of action.

A FRASER-MOODIE

Consultant in Accident and Emergency Medicine D HUGHES

Registrar in Accident and Emergency Medicine, Emergency Departmen Derbyshire Royal Infirmary London Road, Derby DE1 $2 Q Y$

\section{Foreign body removal from children's noses}

EDITOR,-Nasal foreign bodies are a common problem in children attending accident and emergency departments and general practice surgeries. The cooperation required is often not forthcoming as most patients are under 5 years. Instrumentation requiring restraint, sedation, or even general anaesthesia are frequently necessary. This approach may be avoided by using the positive pressure technique described below, which can be used by the parent to quickly and safely remove a nasal foreign body. ${ }^{1-3}$

A history is taken from the parent and child to determine the nature of the foreign body and which nostril is involved. If the child is cooperative, the nares can be examined to locate the foreign body but this is not absolutely necessary if there is a clear indication of which nostril is involved.

Parents are instructed to sit the child on their knee, with the nostril containing the for- eign body closest to the parent. The parent should then close the "patent" nostril using a finger and tell the child that they are going to give them "a great big kiss". Parents should then seal their lips around the child's and deliver a short sharp puff of air. The foreign body is usually extruded with one puff. It is worth holding a paper tissue close by as the foreign body is usually well covered with mucus. If this is unsuccessful several more attempts can be made without any harm. Each additional "puff" may dislodge the foreign body a little further.

The author has used this technique in over 20 cases with no failures or complications. Various objects including beads, peas, pieces of bread, and even a rabbit dropping have been removed successfully. The time from insertion of the foreign body to removal by the technique does not appear to be a factor.

Despite the simplicity of this technique it seems little used. It is easily taught to parents and can be undertaken in any setting. Use of restraints, anaesthesia, and instrumentation in frightened and distressed children can be avoided.

SARAH SCHOFIELD Principal in General Practice, North Baddesley Health Centre Norton Welch Close, Fleming Avenue, North Baddesley, Southampton SO52 9EP

1 Backlin SA. Positive pressure technique for nasa foreign body removal in children. Ann Emer Med 1995;25:554-5.

2 Werman HA. Removal of foreign bodies of the nose. Emerg Med Clin North Am 1987;May: 253-63.

3 Cohen HA, Goldberg E, Thorev Z. Removal of nasal foreign bodies in children. Clin Pediat (Phila) 1993;32:192.

\section{Alcohol intoxication}

EDrToR,-I read with great interest the recent letters by Tovey $e t a l^{l}$ and Denning ${ }^{2}$ regarding the alcohol content of some proprietary paediatric medications and commercially available mouthwashes. Previously I was able to highlight the potential danger to children of perfumed body sprays, ${ }^{3}$ which commonly have an alcohol content of between $70-80 \%$. A child attended the accident and emergency department having consumed the contents of a "tangerine dream" perfumed body spray. She was admitted, but fortunately came to no harm. The presentation of this brand of perfumes was extremely suggestive of a soft drink. No warning was displayed on the containers, which could easily be opened by a child. This article was picked up by several newspapers and a parenting magazine. I subsequently noticed that the company selling these products had withdrawn them from their shelves. The enormous power of the media should be harnessed whenever possible in these and similar areas to protect the most vulnerable in society.

DAVID MOORE

Specialist Registrar in Accident and Emergency Medicine, Accident and Emergency Departmen Norfolk and Norwich Hospital, Brunswick Road, Norwich NR1 $3 S R$

1 Tovey C, Rana PSJB, Anderson DJ. Alcohol intoxication in a toddler. J Accid Emerg Med intoxication in

2 Denning Z. More alcohol. J Accid Emerg Med 1998;15:70.

3 Moore $D$, Gronow $R$, McCabe $M$. Smal children may consume perfumed body spray after mistaking them for soft drinks. BMJ 1996; 313:757.

\section{Fitness to drive}

EdrToR,-Each year there are more than 15 million visits to $U K$ accident and emergency (A\&E) departments. ${ }^{1}$ Many sustain injuries, suffer from medical conditions, or have had drugs administered to them that temporarily impair the patient's ability to drive safely. Yet it is rare to hear discussion of this issue with the patient. A small prospective audit of 21 patients of driving age who were discharged from the department after the application of a limb plaster failed to demonstrate any written advice as to whether they should drive or not.

It is clear that a doctor has a legal duty to warn those patients whose driving may be impaired not to do so and to record this in the notes. ${ }^{2}$ Patients who fail to take the advice of the doctor will probably invalidate their insurance. ${ }^{3}$ The responsibility for the provision of advice for this patient group lies with each A\&E department. "Suitability to drive" is now covered in the senior house officer teaching programme.

$M$ J CLANCY P G POCKNEY Emergency Department Level C, Southampton General Hospital Tremona Road, Southampton SO16 6YD

1 Audit Commission. By accident or design. London: Audit Commission, 1995.

2 Montague A. Legal problems in emergency medicine. Oxford: Oxford University Press, 1996.

3 Giddins GEB, Hammerton A. Doctor, when can I drive? A medical and legal view of the implications of advice on driving after injury or operation. Injury 1996;27:495-7.

\section{Corrections}

Out of hospital cardiac arrest and associated injury by Andrew I Jones, $M$ James Stuart, Alastair J Gray $(\mathcal{A}$ Accid Emerg Med 1998;15:191-2). We regret that because of a production problem the affiliations of the authors of this paper were omitted. They are as follows:

North Tyneside Health Care NHS Trust, Accident and Emergency, North Tyneside General Hospital, Rake Lane, North Shields, Tyne and Wear NE29 8NH A I Jones

Department of Emergency Medicine, Manchester Royal Infirmary M J Stuart

Department of Accident and Emergency, Stepping Hill Hospital, Stockport

A J Gray

Correspondence to: Dr Jones, Consultant.

$\star \star \star \star$

Implementing the Ottawa ankle rules in an Asian accident and emergency medicine department. What potential for saving? $(\mathcal{F}$ Accid Emerg Med 1998;15:132). We regret that there was a spelling error in the first author's name: this should have been Rainer rather than Stainer. 